\title{
Dynamique de l'occupation du sol autour des sites miniers le long du gradient urbain-rural de la ville de Lubumbashi, RD Congo
}

\author{
Yannick Useni Sikuzani ${ }^{(1)}$, Sylvain Boisson ${ }^{(2)}$, Sylvestre Cabala Kaleba ${ }^{(1)}$, \\ César Nkuku Khonde ${ }^{(3)}$, François Malaisse ${ }^{(2)}$, Jean-Marie Halleux ${ }^{(4)}$, Jan Bogaert ${ }^{(2)}$, \\ François Munyemba Kankumbi ${ }^{(1)}$ \\ (1) Université de Lubumbashi. Faculté des Sciences Agronomiques. Unité Écologie, Restauration Écologique et Paysage. \\ 1825 Lubumbashi (RD Congo).E-mail : SikuzaniU@unilu.ac.cd \\ (2) Université de Liège - Gembloux Agro-BioTech. Unité Biodiversité et Paysage. BE-5030 Gembloux (Belgique). \\ (3) Université de Lubumbashi. Faculté des Lettres et Sciences Humaines. Département des Sciences Historiques. 1825 \\ Lubumbashi (RD Congo). \\ (4) Université de Liège. Service de Géographie Économique. BE-4000 Liège (Belgique).
}

Reçu le 8 février 2019, accepté le 13 novembre 2019, mis en ligne le 25 novembre 2019.

Cet article est distribué suivant les termes et les conditions de la licence CC-BY (http://creativecommons.org/licenses/by/4.0/ deed.fr)

Description du sujet. À Lubumbashi, la croissance spatiale urbaine rapide (plus de $5 \%$ par an) et non planifiée concourt à des changements importants de l'occupation du sol, en particulier autour des sites miniers où de fortes concentrations de métaux lourds dans les sols présentent d'énormes conséquences environnementales et sanitaires.

Objectifs. La présente étude vise à évaluer le processus d'anthropisation des paysages autour des sites miniers de la ville de Lubumbashi de 1989 à 2014.

Méthode. À partir de l'approche cartographique couplée aux outils d'analyse paysagère, les tendances évolutives de l'occupation du sol ont été quantifiées à travers la proportion des classes dans des zones tampons comprises entre 0-500 $\mathrm{m}$, 500-1000 m, 1000-1500 m, 1500-2000 m et 2000-2500 m des sites miniers.

Résultats. La périphérie des sites miniers a enregistré une expansion rapide du bâti, suivie d'une régression des sols nus et de la végétation, essentiellement lors de la période qui a suivi la libéralisation du secteur minier en 2002. Cet effet est nettement plus marqué autour des sites miniers urbains. Ces perturbations, qui sont dues à la croissance démographique rapide, s'amplifient avec le temps et sont perceptibles au sein de toutes les classes de distance aux sites miniers.

Conclusions. L'anthropisation des paysages autour des sites miniers pourrait mener à une saturation foncière susceptible d'amplifier la dégradation environnementale et l'exposition humaine aux métaux lourds.

Mots-clés. Facteurs anthropogènes, dégradation des terres, sol contaminé, développement urbain, télédétection/SIG, restauration couverture végétale.

Land cover dynamics around mining sites along urban-rural gradient of Lubumbashi city (DR Congo)

Description of the subject. In Lubumbashi city, rapid (more than 5\% per year) and unplanned spatial urban growth is contributing to important land cover changes, especially around mining sites where high concentrations of heavy metals in soils have enormous environmental and health consequences.

Objectives. This study aimed to evaluate the landscape anthropization process around the mining sites of Lubumbashi city from 1989 to 2014.

Method. Using a mapping approach and the application of landscape ecology analysis tools, land cover changing trends were quantified through the percentage of landscape in the buffer zone, located at 0-500 m, 500-1,000 m, 1,000-1,500 m, 1,5002,000 $\mathrm{m}$ and 2,000-2,500 $\mathrm{m}$ from the mining sites.

Results. The periphery of mining sites registered a rapid built-up expansion accompanied by bare soil and vegetation regression, particularly after the liberalization of the mining sector in 2002. This effect was much more pronounced around mining sites located in the urban zones. These disturbances, which were due to rapid population growth, increased over time and were observable within all the studied distances from the mining sites. 
Conclusions. The anthropization of the landscape around the mining sites could lead to land saturation, and is likely to amplify environmental degradation and human exposure to heavy metals.

Keywords. Anthropogenic factors, land degradation, polluted soil, urban development, remote sensing/GIS, revegetation.

\section{INTRODUCTION}

Les activités minières constituent des sources d'avantages socio-économiques (Ettler, 2016 ; Maconachie, 2012). Depuis le début du $21^{\mathrm{e}}$ siècle, elles s'intensifient dans l'hémisphère sud en parallèle avec l'urbanisation et la croissance démographique (Gwebu, 2012). Toutefois, elles conduisent fréquemment à de profonds changements environnementaux et paysagers tels que la pollution des sols et des eaux, la dégradation des terres et la déforestation (Joshi et al., 2006 ; Musa et al., 2011 ; Panagos et al., 2013 ; Petropoulos et al., 2013 ; Khalil et al., 2014). Il arrive qu'elles constituent une menace pour la santé humaine par exposition aux composés toxiques (Leteinturier et al., 2001 ; Tembo et al., 2006 ; Panagos et al., 2013 ; Ettler, 2016). Cette situation est particulièrement problématique dans les pays en développement où la concentration totale en métaux lourds est généralement beaucoup plus élevée dans les sols autour des sites miniers comparativement à celle enregistrée dans les pays développés (Narendrula et al., 2012), en raison des variations dans le niveau de développement socio-économique et de rigueur dans l'application de la règlementation environnementale (Mwitwa et al., 2012).

En Afrique centrale, l'arc cuprifère, région minière que se partagent la Zambie et la RD Congo, est caractérisé par la présence de gisements de cuivre et de cobalt parmi les plus importants au monde, dont l'exploitation industrielle remonte à plus d'un siècle (Leblanc \& Malaisse, 1978 ; Leteinturier et al., 1999). Dans cette région, les carrières où sont extraites les roches sont situées en périphérie des villes, tandis que le traitement est généralement réalisé dans des usines localisées au sein des villes, notamment à Lubumbashi où ces activités ont été amplifiées depuis la libéralisation du secteur minier en 2002 (Mpundu et al., 2014). Ce phénomène a accentué une dynamique économique et un déplacement massif des populations vers Lubumbashi (Nkuku \& Rémon, 2006), la croissance démographique n'étant malheureusement pas contrôlée. Les demandes en logement sans cesse croissantes qui en résultent sont responsables d'une extension spatiale urbaine rapide et incontrôlée (Useni et al., 2018), tandis que les responsables de la gestion urbaine ne peuvent anticiper ou gérer de manière rigoureuse le zonage urbain (Groupe Huit, 2009 ; Halleux, 2017).

Par conséquent, les sites miniers (sites d'extraction de roches et/ou de traitement de minerais) sont juxtaposés aux habitations (Vranken et al., 2013).
Pourtant, autour de ces sites, la pollution des sols qui résulte de l'exploitation minière a entrainé l'apparition de surfaces dénudées qui présentent des concentrations anormalement élevées en métaux lourds (Narendrula et al., 2012 ; Mees et al., 2013 ; Pourret et al., 2016), mais dont la gestion n'est pas correctement mise en œuvre (Amisi et al., 2008; Munyemba et al., 2008 ; Mwitwa et al., 2012 ; Vranken et al., 2013). Consécutivement, il a récemment été démontré que les habitants des zones situées en périphérie des sites miniers présentaient dans leurs urines des teneurs en cobalt et en cuivre supérieures à la limite autorisée par l'Organisation Mondiale de la Santé (Banza et al., 2009 ; Cheyns et al., 2014). Devant une telle situation, la présence d'un couvert végétal, formé par les espèces de la flore cupro-cobalticole notamment, permet de réduire la dispersion des polluants dans les quartiers environnants en limitant leur transport par érosion hydrique et éolienne (Shutcha et al., 2015).

Il s'avère urgent de comprendre la dynamique spatio-temporelle de l'anthropisation des paysages autour des sites miniers afin de formuler des propositions d'atténuation des impacts environnementaux et sanitaires. Pour cette raison, à travers les outils d'analyse paysagère appuyée par les techniques de la télédétection et des systèmes d'information géographique, la présente étude évalue la dynamique de l'occupation des sols autour des sites miniers de la ville de Lubumbashi de 1989 à 2014, afin de déduire l'état de leurs composantes physique, écologique et sociale (Bogaert \& Mahamane, 2005). Il s'agira de vérifier l'hypothèse selon laquelle, au fil du temps, la faible disponibilité foncière aurait conduit à une forte compétition et pression foncière, avec pour conséquence la réduction de la couverture végétale et l'extension urbaine sur les sols dénudés dans les zones moyennement proches des sites miniers.

\section{MÉTHODOLOGIE}

\subsection{Zone d'étude et sélection des sites miniers}

La zone d'étude, qui intègre la ville de Lubumbashi et sa périphérie, est située dans la province du HautKatanga au sud-est de la RD Congo. Elle couvre une superficie de $1632,4 \mathrm{~km}^{2}$ et est située entre $11^{\circ} 27^{\prime}$ ' et $11^{\circ} 47^{\prime} \mathrm{S}$ et entre $27^{\circ} 19^{\prime}$ et $-27^{\circ} 40^{\prime} \mathrm{E}$ (Figure 1). Elle est située sur une surface d'aplanissement (plateau), accidentée par quelques collines d'orientation nordouest sud-est et de faible dénivellation, dont l'altitude 


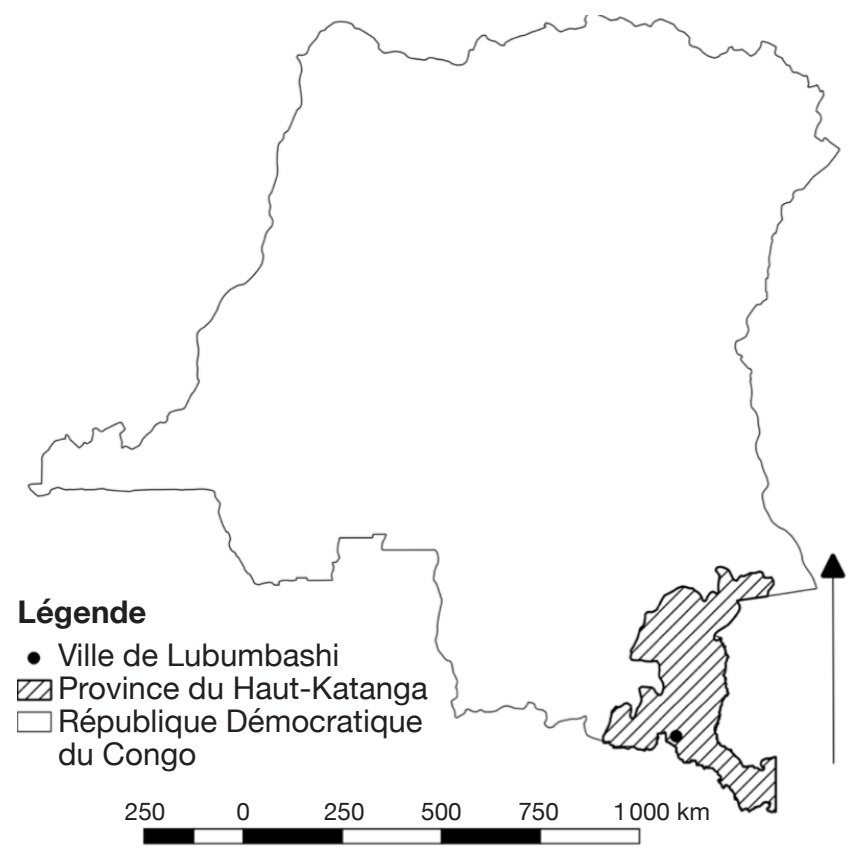

Figure 1. Localisation de la ville de Lubumbashi dans la province du Haut-Katanga, au Sud-Est de la RD Congo - Location of Lubumbashi city in the Upper Katanga province, south-east part of the Democratic Republic of Congo.

générale oscille entre 1200 et $1300 \mathrm{~m}$ (Leblanc \& Malaisse, 1978). Son climat est caractérisé par une saison de pluies, de novembre à mars, et une saison sèche, de mai à septembre ; avril et octobre constituent des mois de transition entre ces deux saisons. Les valeurs moyennes annuelles de précipitations, de température et d'humidité relative sont de l'ordre de $1270 \mathrm{~mm}, 20^{\circ} \mathrm{C}$ et $66 \%$ respectivement (Malaisse, 2010). Les vents dominants soufflent principalement du sud-est vers le nord-ouest avec de légères oscillations (Bruneau \& Pain, 1990 ; Leteinturier et al., 1999). Ils contribuent à la dispersion des métaux lourds sur les sols de Lubumbashi (Vranken et al., 2013), dont les plus dominants sont les ferralsols (Mujinya et al., 2014). La végétation dominante dans la plaine de Lubumbashi, dans un état fragmenté en raison de diverses activités anthropiques (Munyemba \& Bogaert, 2014 ; Useni et al., 2017), était la forêt claire de type miombo (Malaisse, 2010). Actuellement, cette forêt claire est remplacée par des savanes herbeuses et boisées. L'agriculture, l'élevage résidentiel, les services, l'exploitation minière et le commerce sont les principales activités d'une population qui a approché 2 millions d'habitants en 2014 (UN-Habitat, 2014).

Tous les sites miniers présents le long du gradient urbain-rural de la ville de Lubumbashi ont été retenus sur la base de leur période de création et de leur position (Figure 2) :

- la carrière de Congo Dongfang Mining (CDM) créée en 1996 ;
- l'usine de Congo Dongfang Mining (CDM) créée en 2010 ;

- les carrières du complexe Ruashi Mining, créé ca 2003 et la mine de l'Étoile, créée en 1910 ;

- l'usine de Chemical of Africa (CHEMAF) créée en 2002 ;

- le complexe industriel Société de Terril de Lubumbashi (STL), créé en 1997 et les Usines de la Gécamines (UL-Gécamines), créées en 1910 ;

- l'usine de la Société Minière du Katanga (SOMIKA), créé en 2001.

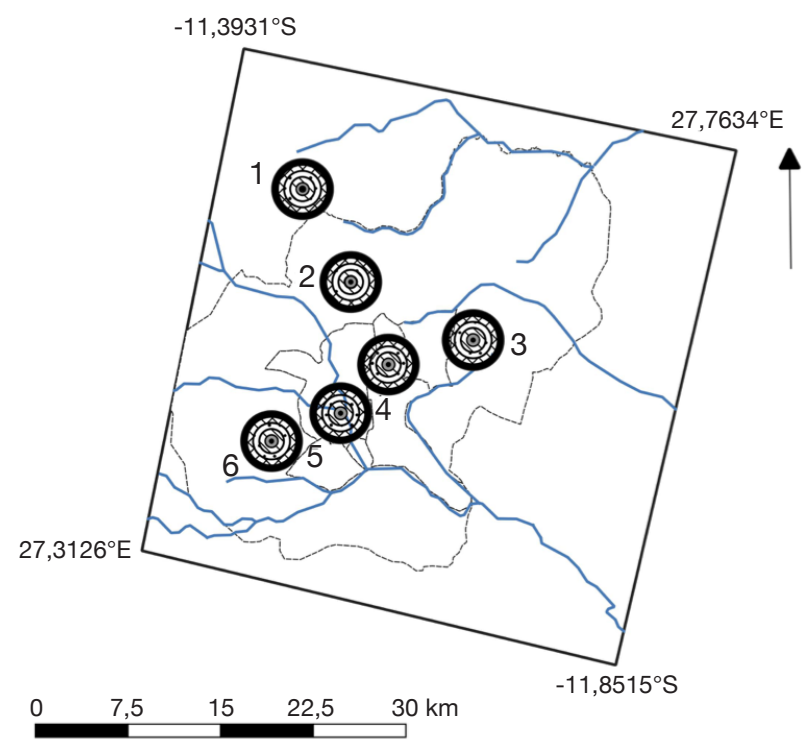

\section{Légende}

- Sites miniers

Zone tampon 0-500 m

Zone tampon 500-1000 m

... Zone tampon 1000-1500 m

Zone tampon 1500-2000 m

Zone tampon 2000-2500 m

Cours d'eau

Limites de la ville et de ses communes Zone d'étude

Figure 2. Dispositif pour le calcul de la proportion des classes d'occupation du sol à égales distances des sites miniers. Des surfaces ont été délimitées par des zones tampons dans les rayons de 0-500 m, 500-1000 m, 1000$1500 \mathrm{~m}, 1500-2000 \mathrm{~m}$ et $2000-2500 \mathrm{~m}$ de la carrière CDM [1], de l'usine CDM [2], du complexe minier Ruashi Mining et la mine de l'Étoile [3], de l'usine de CHEMAF [4], du complexe industriel UL-Gécamines [5] et de l'usine SOMIKA [6] - Device for calculating the land cover proportion at equal distances from mining sites. Areas have been demarcated by buffer zones in the 0-500 m, 500$1,000 \mathrm{~m}, 1,000-1,500 \mathrm{~m}, 1,500-2,000 \mathrm{~m}$ and 2,000-2,500 m radii of CDM quarry [1], CDM industry [2], Ruashi Mining and 'la mine de l'Étoile' complex [3], CHEMAF industry [4], STL and UL and Gécamines complex [5] and SOMIKA industry [6]. 
Il s'avère que les sites susmentionnés ont été créés entre 1996 et 2010, excepté l'usine de la Gécamines et la mine de l'Étoile dont l'existence remonte autour de 1910.Les usines sont situées en zone urbaine (CHEMAF, UL-Gécamines) et périurbaine (CDM et SOMIKA). Les carrières, pour leur part, sont situées dans la zone périurbaine suivant les critères morphologiques établis par l'arbre de décision d'André et al. (2014) et sont à ciel ouvert. Dans la zone urbaine, les surfaces bâties dominent ou sont continues et l'utilisation du sol est essentiellement résidentielle. En revanche, dans la zone périurbaine, les surfaces bâties ne dominent pas ou sont discontinues, il n'y a pas de zonation explicite des utilisations du sol (André et al., 2014).

\subsection{Matériel et méthodes}

Choix d'images satellitaires. La zone d'étude a été isolée sur des images SPOT prises sur la période de 1989-2014, à six dates différentes et avec un pas de temps de 3 à 6 ans. Ces images, issues des capteurs HRG2 (16/07/2002, 18/06/2008 et 20/06/2014; $10 \mathrm{~m}$ de résolution), HRV2 (12/07/1989 et 29/08/1996; $20 \mathrm{~m}$ de résolution) et HRVIR2 (13/08/2005; $20 \mathrm{~m}$ de résolution), ont été choisies sur la base de leur disponibilité et traitées avec les logiciels ENVI 4.5 et ArcGIS 10.1. Il convient de rappeler que les images SPOT utilisées couvrent deux périodes vis-à-vis de la libéralisation du secteur minier en RD Congo en 2002 : avant (1989 et 1996) et après (2005, 2008 et 2014). Les sites miniers, les points de contrôle au sol et les zones d'entrainement ont été collectés à l'aide d'un GPS (Garmin 64st, précision $\pm 3 \mathrm{~m}$ ).

Pré-traitement d'images. Toutes les images acquises avaient déjà subi une correction radiométrique par le fournisseur. Le seul prétraitement qui est réalisé a consisté en un géoréférencement dans le référentiel UTM Zone $35 \mathrm{~s}$ basé sur l'ellipsoïde de référence WGS 84, excepté pour l'image de 2008 rectifiée par le fournisseur. Pour cette raison, l'image de 2008 a servi de référence à l'orthorectification des autres images SPOT retenues, avec une précision géométrique inférieure à 0,5 pixel (Mas, 2000). Pour homogénéiser la taille de pixels à $10 \mathrm{~m}$ de résolution, un ré-échantillonnage cubique a été appliqué car il conserve les valeurs radiométriques des images (Mama et al., 2013).

Classification d'images. Une composition colorée fausse couleur, dans l'ordre proche infrarouge, rouge et vert, a été réalisée pour chacune des images SPOT retenues afin de mieux discriminer la végétation et les occupations du sol anthropiques (Barima et al., 2009). Quinze classes d'occupation du sol ont tout d'abord été créées suite à une classification non supervisée de l'image SPOT de 2014, ce qui a permis de regrouper, sur la base des connaissances de la zone d'étude et d'anciennes cartes, les occupations du sol similaires. L'occupation du sol a, par la suite, été identifiée et cartographiée, suivant la nomenclature AFRICOVER, sur chacune des images composites par une approche de classification supervisée en utilisant l'algorithme du maximum de vraisemblance. Cet algorithme utilise les statistiques des zones d'entrainement pour calculer la probabilité maximale d'appartenance des pixels aux occupations du sol prédéfinies (Caloz \& Colet, 2001). Pour cette raison, des zones d'entrainement homogènes sous forme de polygone ont été collectées pour chaque classe d'occupation du sol lors des missions de terrain. Au départ, ces zones comprenaient neuf classes d'occupation du sol qui ont été finalement regroupées pour les besoins de l'étude. Les classes d'occupation du sol retenues dans notre étude ont été le bâti, le sol nu (le terrain non bâti et sans végétation), la végétation (la forêt, les marécages, les champs, et les savanes herbeuses et boisées) et les autres occupations du sol (les plans d'eau, le terril, les zones brulées). Les autres occupations du sol ont été exclues de la suite des analyses au regard de leur proportion relativement faible dans le paysage.

Évaluation de la qualité de la classification et cartographie de la zone d'étude. Des points de référence (entre 40 pour les autres occupations du sol et 480 pour la classe de végétation qui était composée de plusieurs types de végétation) ont été aléatoirement et minutieusement sélectionnés sur le terrain au cours de la saison sèche (mai-aout) de l'année 2015 afin d'apprécier la qualité de la classification réalisée. Pour chaque classe d'occupation du sol, le nombre de points était fonction de sa représentativité sur l'ensemble de la zone d'étude, de sa superficie et de son intérêt pour cette étude. Ces points de référence ont servi à la validation de toutes les images SPOT acquises entre 1989 et 2014. La qualité de la classification a été appréciée à travers la précision globale et le coefficient Kappa qui comparent les données de télédétection aux données de référence sur terrain (Landis \& Koch, 1977). Un filtre majoritaire a été appliqué afin de résorber les pixels isolés. Les images SPOT classifiées ont été vectorisées afin d'extraire les polygones relatifs aux occupations du sol retenues. Le traitement cartographique a permis la production de six cartes d'occupation du sol.

Analyse de la dynamique d'occupation du sol. Afin d'établir les relations entre la configuration des paysages et les processus écologiques, il est utile de décrire ces structures en termes quantifiables à travers une série d'indices (Bogaert \& Mahamane, 2005). C'est la proportion des classes dans le paysage qui a été retenue dans le présent contexte. Cet indice a été calculé à l'échelle de la zone d'étude et dans des zones tampons 
comprises entre 0-500 m, 500-1000 m, 1000-1500 m, 1500-2000 m et 2000-2500 m du centre géométrique (centroïde) des sites miniers sélectionnés (Figure 2). Ces distances ont été fixées de manière aléatoire dans la zone identifiée comme celle de forte exposition humaine aux métaux lourds à Lubumbashi par Banza et al. (2009). La bande de 0-500 $\mathrm{m}$ intègre aussi les constructions appartenant aux sites miniers retenus. La détermination des distances a été réalisée afin d'apprécier la dynamique de l'occupation du sol plus près $(0-500 \mathrm{~m})$, près $(500-1000 \mathrm{~m})$, moyennement près $(1000-1500 \mathrm{~m})$, loin $(1500-2000 \mathrm{~m})$ et très loin $(2000-2500 \mathrm{~m})$ des sites miniers. Pour évaluer l'ajustement de données à la loi normale, le test de Kolmogorov-Smirnov a été appliqué. Ce test a été appliqué en raison de l'importance de l'échantillon. Par la suite, le test non paramétrique de KruskallWallis a été effectué pour évaluer l'effet année et l'effet distance sur la proportion des occupations du sol autour des sites miniers. Enfin, la proportion des occupations du sol a été comparée entre les sites miniers urbains et périurbains à travers le test de Mann-Whitney. Les analyses statistiques ont été réalisées grâce au logiciel $\mathrm{R}$, avec une valeur seuil de probabilité fixée à $5 \%$.

\section{RÉSULTATS}

\subsection{Précision de la classification supervisée et cartographie}

$\mathrm{Au}$ terme de la classification supervisée, la matrice de confusion a donné des valeurs de précision globale variant de $88,9 \%$ à $98,2 \%$ et celles du coefficient Kappa de 87,1 \% à 97,9\%. Globalement, la discrimination entre les classes d'occupation du sol a été statistiquement fiable (Tableau 1 ; Annexe 1).

\section{2. Évolution de l'occupation du sol dans la zone d'étude}

L'analyse des cartes a révélé des changements considérables dans la zone d'étude, marqués par une évolution spatiale progressive ou régressive des classes d'occupation du sol de 1989 à 2014. De manière générale, l'évolution de l'occupation du sol a été caractérisée, entre 1989 et 2014, par une expansion rapide du bâti et une régression de la couverture de sol nu et de la végétation (Tableau 2). L'évolution spatiale de l'anthropisation du paysage a été particulièrement forte après la libéralisation du secteur minier (entre 2005 et 2014). En effet, cette libéralisation a conduit à une dynamisation de l'activité minière qui a ainsi renforcé l'attractivité de la ville dont la sphère d'influence a dépassé ses limites administratives stricto sensu.

La proportion du bâti a quadruplé dans le paysage de $1989(5 \%)$ à 2014 (19\%) (Figure 3). L'expansion du bâti est observée dans toutes les directions autour de la ville, en entrainant une importante dégradation de la végétation, mais aussi une diminution des sols nus. En effet, la proportion des sols nus a été divisée par 3 en 25 ans (1989-2014), en passant de 1,5\% en 1989 à $0,5 \%$ en 2014. Malgré cela, il convient de noter que la proportion maximale de près de $3 \%$ a été enregistrée au cours de la période qui a précédé la libéralisation du secteur minier (en 2005). La classe de végétation, matrice du paysage, au cours des six dates, étudiées, a enregistré une régression de $15 \%$ en 25 ans, d'autant plus que sa proportion dans le paysage a chuté à près de $80 \%$ en 2014, comparativement à la valeur initiale de près de $93 \%$ en 1989 .

\subsection{Dynamique de l'occupation du sol autour des sites miniers}

Autour des sites miniers (Figure 4), il existe une différence significative au niveau de la proportion des classes d'occupation du sol au fil du temps $(p<0,05)$. La proportion du bâti a fortement augmenté au cours de six dates retenues. Pour l'ensemble des terrains situés à moins de $2500 \mathrm{~m}$ des sites miniers analysés, la proportion du bâti est passée de $21 \%$ en 1989 à $67 \%$ en $2014(\mathrm{H}: 52,93$; dl : 5 ; $p: 0,000)$. Si la proportion de sol nu a quasiment doublé autour des sites miniers entre 2002 et 2005, la période comprise entre 2005 et 2014 a été marquée par une division de sa proportion par $7(\mathrm{H}: 21,79 ; \mathrm{dl}: 5 ; p: 0,001)$. En effet, sur cet intervalle de temps, la proportion des sols nus est passée de $\pm 14 \%$ à $\pm 2 \%$. Enfin, la proportion de végétation a été divisée par environ 3 autour des sites

Tableau 1. Synthèse des indices illustrant la fiabilité de la précision des images SPOT classifiées sur la base de l'algorithme du maximum de vraisemblance et à partir de 760 points prélevés sur le terrain. Bâti : 120 points, sol nu : 120 points, végétation : 480 points, autres occupation du sol : 40 points - Synthesis of indices illustrating the accuracy of the classified SPOT images on the basis of the maximum likelihood algorithm, and from 760 ground points. Built-up: 120 points, bare soil: 120 points, vegetation: 480 points, other land covers: 40 points.

\begin{tabular}{lllllll}
\hline Indice & $\mathbf{1 9 8 9}$ & $\mathbf{1 9 9 6}$ & $\mathbf{2 0 0 2}$ & $\mathbf{2 0 0 5}$ & $\mathbf{2 0 0 8}$ & $\mathbf{2 0 1 4}$ \\
\hline Précision globale (\%) & 89,2 & 89,5 & 88,9 & 92,4 & 93,7 & 98,2 \\
Coefficient Kappa (\%) & 87,5 & 87,7 & 87,1 & 91,1 & 92,7 & 97,9 \\
\hline
\end{tabular}


Tableau 2. Composition de l'occupation du sol en 1989, 1996, 2002, 2005, 2008 et 2014 dans la zone d'étude en km² — Land cover composition within the study area in 1989, 1996, 2002, 2005, 2008 and $2014 \mathrm{in} \mathrm{km}^{2}$.

\begin{tabular}{|c|c|c|c|}
\hline \multirow[t]{2}{*}{ Année } & \multicolumn{3}{|c|}{ Type d'occupation du sol } \\
\hline & Végétation & Sol nu & Bâti \\
\hline 1989 & $1503,4(92,1)$ & $22,9(1,4)$ & $80,0(4,9)$ \\
\hline 1996 & $1477,3(90,5)$ & $37,5(2,3)$ & $111,0(6,8)$ \\
\hline 2002 & $1456,1(89,2)$ & $21,2(1,3)$ & $129,0(7,9)$ \\
\hline 2005 & $1416,9(86,8)$ & $50,6(3,1)$ & $151,8(9,3)$ \\
\hline 2008 & $1412,0(86,5)$ & $27,8(1,7)$ & $189,4(11,6)$ \\
\hline 2014 & $1310,8(80,3)$ & $8,2(0,5)$ & $310,2(19,0)$ \\
\hline
\end{tabular}

Les valeurs dans la parenthèse correspondent à la proportion dans la zone d'étude. La somme d'aires de chaque date, en valeur absolue ou relative, ne correspond pas à l'aire totale du paysage car les « autres occupations du sol » ont été exclues des analyses - The values in bracket correspond to the proportion in the study area. The sum of area for each date is not $100 \%$ because "other land cover classes » were excluded from the analyzes.

miniers de 1989 à 2014, d'autant plus qu'elle est passée de $\pm 73 \%$ à $\pm 22 \%$. Il ressort de l'analyse statistique que la proportion de la végétation a diminué au fil du temps entre 1989 et $2014(\mathrm{H}: 14,12 ; \mathrm{dl}: 5 ; p: 0,015)$.

La figure 5 rend compte de l'évolution de l'occupation du sol à différentes classes de distance aux sites miniers entre 1989 et 2014. Autour de ces sites, la proportion du bâti est élevée à proximité et elle diminue légèrement au fur et à mesure qu'on s'en éloigne. Toutefois, l'analyse statistique a révélé que les proportions de bâti sont similaires dans les différentes classes de distance aux sites miniers $(\mathrm{H}: 0,56 ; \mathrm{dl}: 4 ; p: 0,967)$. En revanche, la proportion du bâti est plus élevée autour des sites miniers urbains comparativement aux sites miniers périurbains ( $\mathrm{W}: 21782,0$; $p: 0,0000)$.

Pour le sol nu, on note une augmentation de sa proportion avec l'éloignement aux sites miniers urbains et, à l'inverse, une réduction de sa proportion au fur et à mesure qu'on s'éloigne des sites miniers périurbains. L'analyse statistique a établi que les proportions de sol nu sont similaires entre les différentes classes de distance aux sites miniers (H : 3,44; $\mathrm{dl}: 4 ; p: 0,487)$. Par ailleurs, les proportions de sol nu sont nettement moins élevées autour des sites miniers périurbains comparativement aux sites miniers urbains (W : 22629,0 ; $p: 0,0004$ ).

En ce qui concerne la végétation, sa proportion est plus faible autour des sites miniers urbains que dans la périphérie des sites miniers périurbains (W: 1354,0; $p: 0,0000$ ). L'analyse statistique a révélé que toutes les classes de distances aux sites miniers ont présenté des proportions similaires de végétation $(\mathrm{H}: 0,81 ; \mathrm{dl}: 4 ; p: 0,937)$. Quelle que soit la classe de distance aux sites miniers, la proportion de la végétation a enregistré une régression continue entre 1989 et 2014. Néanmoins, il convient de noter que la régression la plus importante est observée au niveau des sites miniers périurbains.

En synthèse, sur l'ensemble de la période comprise entre 1989 et 2014, le bâti a constitué la matrice

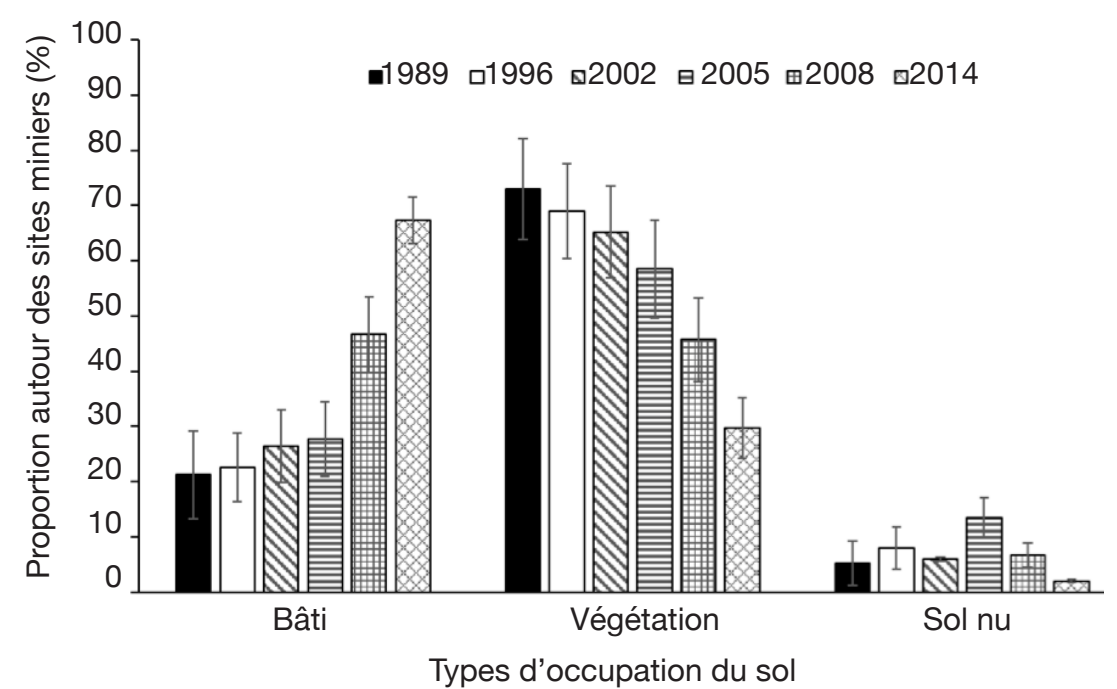

Figure 4. Proportion moyenne des classes d'occupation autour des sites miniers entre 1989 et 2014 - Evolution of the average land cover proportion around mining sites between 1989 and 2014.

Les barres réfèrent à l'écart-type des moyennes de tous les sites miniers étudiés et de toutes les classes de distances aux sites miniers - The bars refer to the standard deviation of the averages of all mining sites studied and all distances classes from mining sites. 

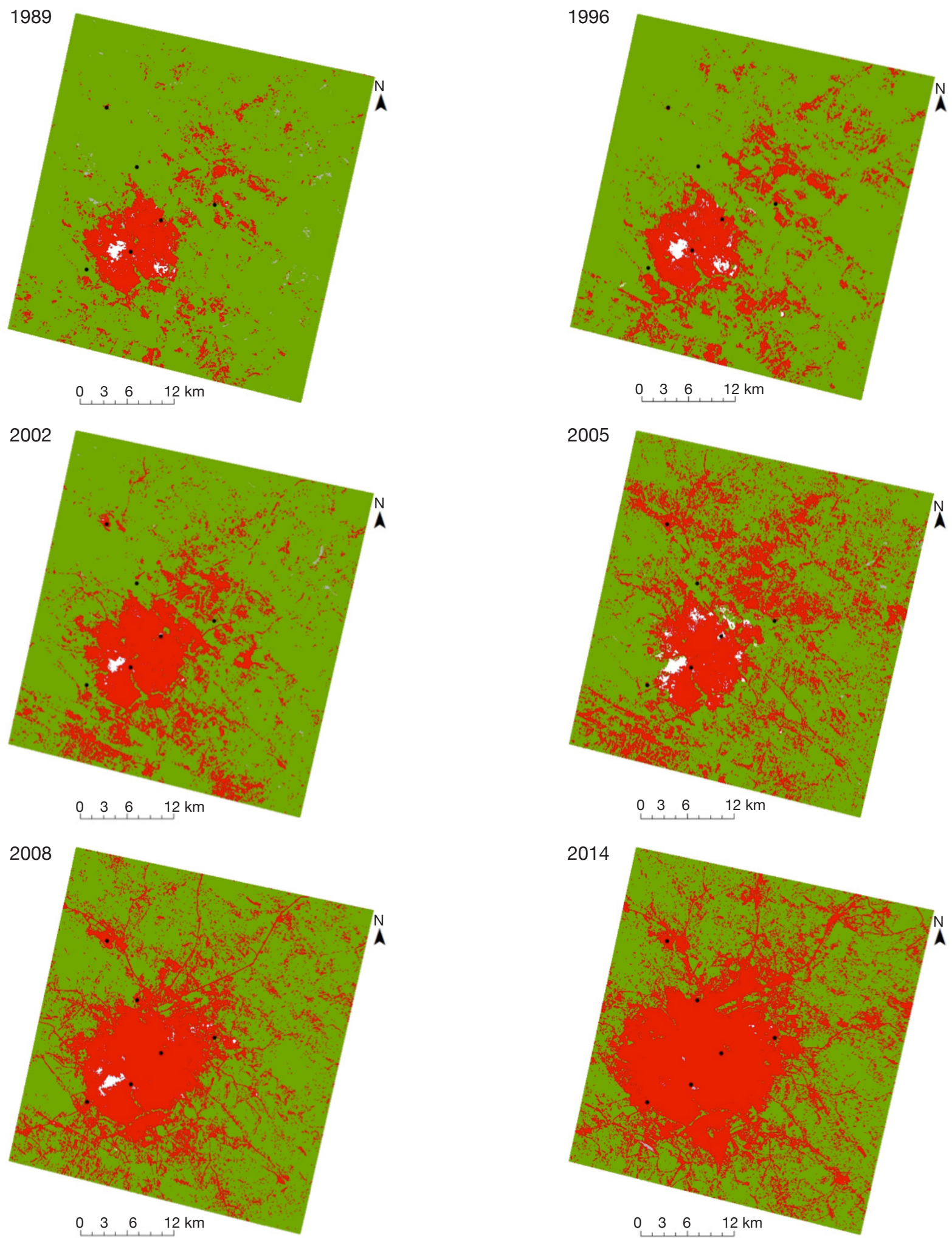

- Site minier $\square$ Bâti $\square$ Sol nu Végétation Autres occupations du sol

Figure 3. Cartes d'occupation du sol de la zone d'étude en 1989, 1996, 2002, 2005, 2008 et 2014 sur base de la classification supervisée des images SPOT. Les six cartes couvrent deux périodes vis-à-vis de la libéralisation du secteur minier en RD Congo en 2002 : avant (1989-1996) et après (2005-2014) - Land cover maps of the study area in 1989, 1996, 2002, 2005 , 2008 and 2014 based on the supervised classification of SPOT images. The six maps cover two periods linked to the DR Congo mining sector's liberalization in 2002: before (1989-1996) and after (2005-2014). 
a

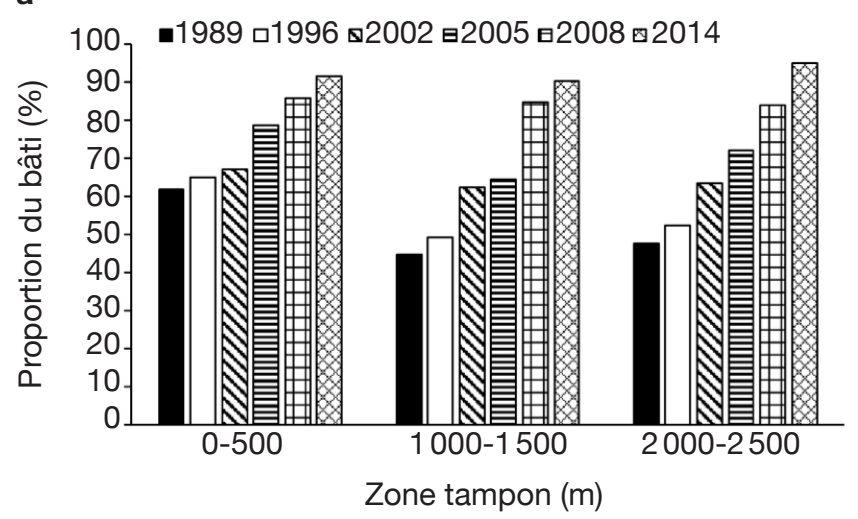

c

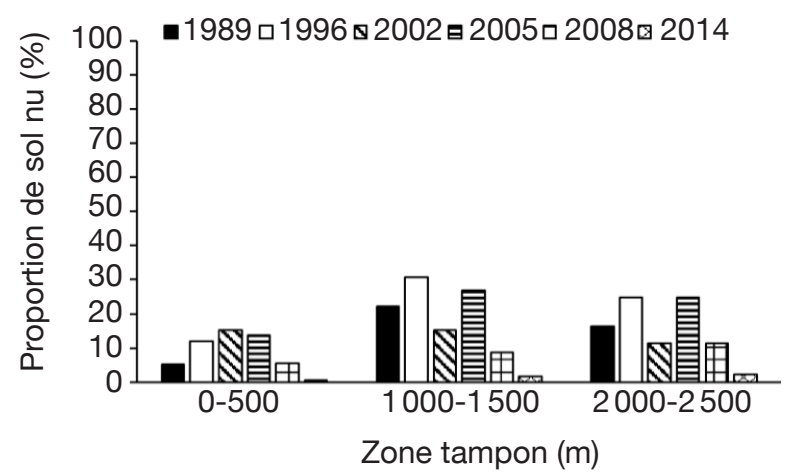

e

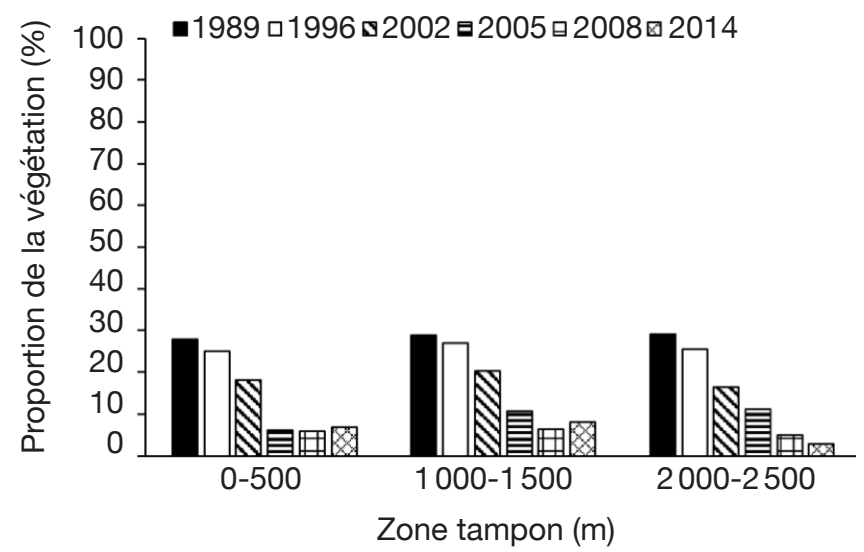

b

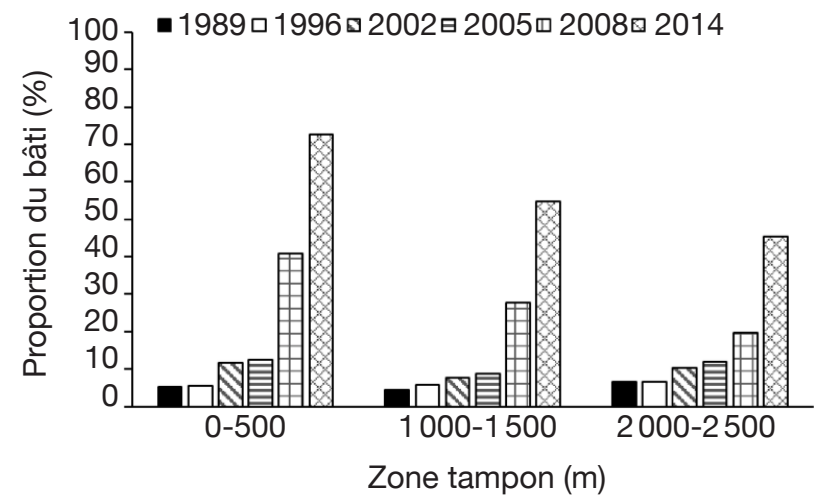

d

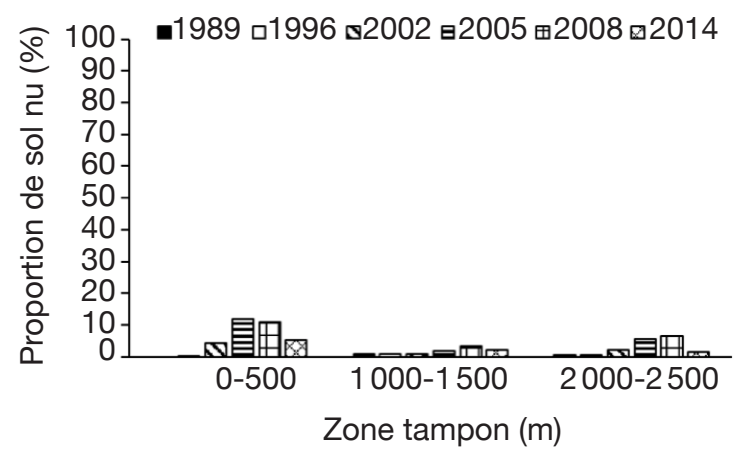

f

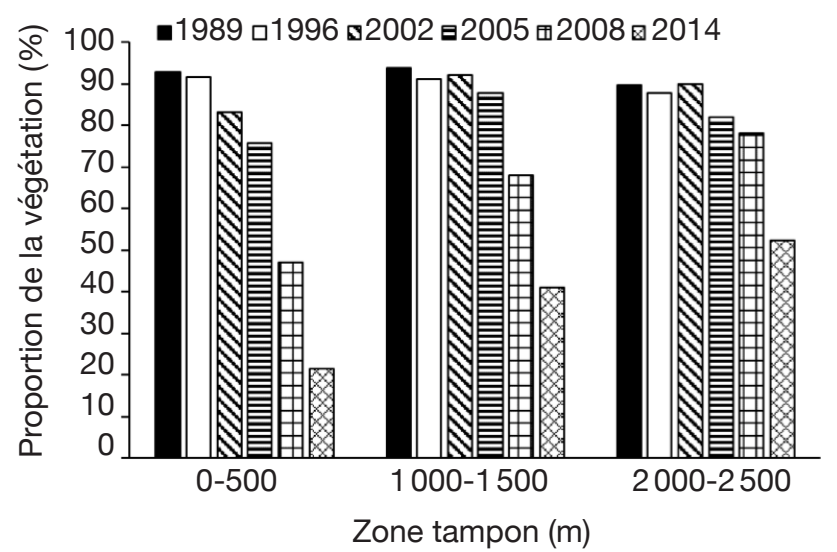

Figure 5. Proportion des classes d'occupation du sol à différentes classes de distances aux sites miniers entre 1989 et 2014. À gauche (a, c et e), l'évolution de la proportion des classes d'occupation du sol autour des sites miniers urbains. L'évolution de la proportion des classes d'occupation du sol autour des sites miniers périurbains est illustrée par les graphiques à droite (b, d et f). Les classes intermédiaires (500-1000 m et 1500-2000 m) ont été exclues sur les graphiques afin d'éviter la redondance d'informations. Les superficies totales des zones tampons retenues correspondent à $0,785 \mathrm{~km}^{2}, 3,925 \mathrm{~km}^{2}$ et $7,065 \mathrm{~km}^{2}$ respectivement pour les distances de 0-500 m, 1000-1500 m et 2000-2500 m des sites miniers - Evolution of land cover proportion at different distances classes from mining sites between 1989 and 2014. On the left (a, $\boldsymbol{c}$ and $\boldsymbol{e}$ ), changes in the land cover proportion around mining sites of the urban zone. The evolution of land cover proportion around mining sites of the peri-urban zone is illustrated by the graphs on the right $(\boldsymbol{b}, \boldsymbol{d}$ and $\boldsymbol{f})$. Intermediate classes $(500-1,000 \mathrm{~m}$ and 1,500 $2,000 \mathrm{~m}$ ) have been excluded on the graphics to avoid redundancy of information. The total areas of the buffer zones selected correspond to $0.785 \mathrm{~km}^{2}, 3.925 \mathrm{~km}^{2}$ and $7.065 \mathrm{~km}^{2}$ for distances of 0-500 m, 1,000-1,500 m and 2,000-2,500 m from mining sites. 
paysagère autour des sites miniers urbains. Par contre, la végétation continue de représenter la matrice paysagère autour des sites miniers périurbains. La périphérie des sites miniers est caractérisée par une importante destruction de la végétation et les espaces dénudés qui en résultent sont envahis par le bâti, une faible tendance à la recolonisation de la végétation n'étant pas à exclure. Ces mutations paysagères pourraient mener à une saturation foncière, considérée comme une occupation complète des espaces ouverts.

\section{DISCUSSION}

\subsection{Méthodologie}

La cartographie et l'analyse de la dynamique de l'occupation du sol font appel à l'application des méthodes de classifications, ce qui nécessite une vérification de leur qualité (Caloz \& Colet, 2001). Dans cette étude, la résolution spatiale assez fine des images utilisées, leur traitement avant classification, le regroupement des classes d'occupation du sol et la connaissance de la zone d'étude ont permis d'en améliorer la qualité, comme l'illustrent les valeurs acceptables du coefficient Kappa (Landis \& Koch, 1977). Malgré quelques critiques formulées sur cet indice (Pontius \& Millones, 2011), son utilisation dans cette étude se justifie par le fait que le nombre de pixels de sites d'entrainementétait similaire pour chaque classe d'occupation du sol pour les différentes dates retenues (Masse et al.,2012).La composition des paysages autour des sites miniers a été appréciée à travers un dispositif à base de zones tampons. Cette approche de détection des dynamiques paysagères est importante pour l'observation d'un phénomène évoluant le long d'un gradient socio-environnemental à partir d'une source. Elle a déjà été appliquée dans plusieurs études (Seto \& Fragkias, 2005 ; Bamba et al., 2010 ; Havyarimana et al., 2018). Dans la présente étude, la limite de $2500 \mathrm{~m}$ autour des sites miniers a permis d'éviter le chevauchement entre sites rapprochés et est comprise dans la zone de risque élevé d'exposition humaine aux métaux lourds (Banza et al., 2009). Quoique l'utilisation de plusieurs indices soit généralement requise pour quantifier la structure spatiale des paysages (Bogaert \& Mahamane, 2005), seule la proportion des classes dans le paysage s'est révélée pertinente pour dégager les tendances évolutives de l'occupation du sol dans la présente étude. Cet indice a permis de caractériser avec succès la déforestation autour des villes/routes en RD Congo (Bamba et al., 2010) et des camps des déplacés au Burundi (Havyarimana et al., 2018). Néanmoins, en fonction de la complexité du phénomène étudié, le choix d'un indice unique n'est pas toujours la meilleure solution.

\subsection{Dynamique de l'occupation du sol autour des sites miniers urbains et périurbains}

À Lubumbashi, la reprise des activités minières depuis la promulgation du Code minier en 2002 a entrainé des changements sociaux importants, notamment une croissance démographique rapide (Nkuku \& Rémon, 2006). En effet, le taux moyen annuel de croissance démographique est passé de 4,1\% entre 1989 et 2002 à 8,3\% de 2002 à 2014 (UN-Habitat, 2014). La fourniture en logements est apparue comme une question vitale et un des faits les plus marquants de la dynamique paysagère à Lubumbashi. Ceci s'est traduit par une expansion spatiale urbaine rapide (Groupe Huit, 2009; Useni et al., 2018), qui se produit en échappant au contrôle des pouvoirs publics (Trefon \& Cogels, 2007). Les bénéfices générés par l'exploitation des ressources minières et leurs implications sociales associées sont les principaux facteurs de l'urbanisation rapide dans plusieurs villes africaines (Bryceson \& Mackinnon, 2012 ; Gwebu, 2012 ; Maconachie, 2012 ; Mususa, 2012 ; Rodrigues \& Tavares, 2012).

La pénurie de l'offre foncière constructible a poussé certains ménages à s'installer sur les rares terrains vides situés à proximité des sites miniers, car coutant moins chers. Pourtant, autour de ces sites, les actions anthropiques ont contribué à éparpiller les polluants dont les dépôts s'expriment d'une part à travers la mise à nu de la surface des sols et, d'autre part, via l'installation des espèces de la flore cupricole (Munyemba et al., 2008). La forte présence des surfaces de sol nu, mise en évidence entre 1989 et 2005, serait attribuée à la destruction du couvert végétal à proximité des sites miniers, probablement en raison des aménagements effectués pour implanter les sites miniers d'une part et, d'autre part, à cause de l'impact de la pollution aux métaux lourds (Leteinturier et al., 2001 ; Tembo et al., 2006 ; Mees et al., 2013). Dans le contexte de cette étude, d'un côté les sols nus autour des sites miniers constituent des terrains faciles à urbaniser. De l'autre, ils sont problématiques pour la santé des populations car considérés non constructibles en raison de leur concentration élevée en métaux lourds (Leblanc \& Malaisse, 1978 ; Bruneau \& Pain, 1990).

Autour des sites miniers, le processus d'urbanisation reste non planifié, informel et fréquemment marqué par des conflits et une insécurité relative à l'utilisation des terres (Weerts, 2015). Cette situation est favorisée par une confusion dans les rôles et responsabilités des acteurs concernant l'urbanisation et la gestion urbaine (Groupe Huit, 2009). Les rares espaces verts susceptibles d'atténuer les effets néfastes de la pollution autour des sites miniers, y compris les espèces de la flore cupricole qui recolonisent spontanément les habitats secondaires (Boisson et al., 2015), sont systématiquement détruits et dégradés 
par l'auto-construction (Leblanc \& Malaisse, 1978 ; Munyemba et al., 2008 ; Vranken et al., 2013). Cette observation correspond à l'évolution de la dynamique paysagère caractéristique de la partie sud du Katanga, où les changements socio-économiques ont affecté la dynamique du paysage (Nkuku \& Rémon, 2006 ; Vranken et al., 2013). Dans un contexte social difficile, les populations se tournent vers les sites miniers pour la recherche d'équipements socio-techniques, mais surtout pour bénéficier de diverses actions sociales posées par les miniers. L'installation de milliers de personnes autour des sites miniers a un impact négatif en termes de dégradation de sols et de la végétation, susceptible d'entrainer une perte de la biodiversité (Vranken et al., 2013 ; Boisson et al., 2015 ; Shutcha et al., 2015). L'urbanisation autour de ces sites contribue ainsi à la détérioration du cadre de vie des populations riveraines et, en raison de la combinaison complexe d'acteurs, elle exacerbe les rivalités tant pour l'espace que pour les ressources (Trefon \& Cogels, 2007 ; Bogaert et al., 2015 ; Weerts, 2015).

\subsection{Implications pour l'aménagement du territoire}

À Lubumbashi, un éventail d'études épidémiologiques a montré une forte exposition humaine aux métaux lourds autour des sites miniers (Banza et al., 2009 ; Cheyns et al., 2014). Les populations riveraines aux sites miniers connaissent bien la situation, puisqu'elles ont identifié des preuves de la pollution aux métaux dans leurs quartiers (Amisi et al., 2008 ; Vranken et al., 2013). En plus, un certain nombre de conflits sociaux, menant à une insécurité foncière persistante, sont régulièrement signalés (Groupe Huit, 2009). En raison de la croissance démographique rapide et de l'absence d'un zonage, faute d'outils pratique et de formation des aménageurs locaux, l'étalement urbain devrait se poursuivre au cours des prochaines décennies en créant de nouvelles zones de contact habitat-sites miniers (Vranken etal.,2013; Munyemba \& Bogaert,2014). Par conséquent, la dépollution des sols nus autour des sites miniers devrait être une priorité dans l'aménagement urbain à Lubumbashi. À cet effet, la phytorémédiation constitue une solution durable, d'autant plus qu'elle permet de lutter contre l'érosion, de réinsérer les sols dénudés dans le paysage et de préserver la biodiversité (Boisson et al., 2015). Une telle approche a déjà donné expérimentalement des résultats prometteurs sur un sol nu pollué à proximité des habitations, dans la partie centrale de la ville de Lubumbashi (Shutcha et al., 2015). Aussi, les plantations d'arbres coupe-vent pourraient s'avérer utile. Cependant, le fait que certains sols contaminés aient été occupés par les habitations rend la phytoremédiation d'autant plus difficile que la décontamination y est prioritaire (Vranken et al.,
2013). Une autre solution, moins couteuse, est de faire en sorte que les habitants ne s'installent pas sur les sols nus potentiellement pollués autour des sites miniers à travers l'application des textes règlementaires qui existent par ailleurs. Pour cela, il convient de planifier la croissance urbaine et viabiliser suffisamment de terrains pour accueillir la population en augmentation (Leblanc \& Malaisse, 1978 ; Bruneau \& Pain, 1990 ; Groupe Huit, 2009 ; Halleux, 2017).

\section{CONCLUSIONS}

La libéralisation du secteur minier en RD Congo a entrainé des changements socio-économiques qui se sont répercutés sur le paysage urbain à Lubumbashi, ainsi que sa zone périphérique. La présente étude a permis de révéler que l'expansion spatiale du bâti dans la zone d'étude a été accompagnée d'une régression importante de sol nu et de la végétation pendant la période allant de 1989 à 2014. Ces perturbations sont plus marquées autour des sites miniers. À proximité de ces sites, l'urbanisation inappropriée se produit sur des terrains potentiellement pollués et entraine en même temps la destruction et la dégradation de la végétation. Ce phénomène, observé au sein de toutes les classes de distance aux sites miniers, est plus inquiétant autour des sites miniers situés en zone urbaine. Si la période d'avant la libéralisation du secteur minier a été caractérisée par une régression modeste de la végétation parallèlement à une relative augmentation de la surface de sol nu, l'urbanisation non planifiée autour des sites miniers a amplifié leur régression durant la période qui a suivi la libéralisation du secteur minier. Autour des sites miniers, les activités anthropiques peuvent aller jusqu'à la rupture de l'équilibre écologique à travers une tendance à la saturation foncière si aucune mesure n'est prise. Cette étude permet d'attirer l'attention des décideurs et aménageurs sur la nécessité d'élaborer des outils efficaces d'aménagement du territoire et de préservation de la végétation afin d'améliorer la santé des populations riveraines des sites miniers.

\section{Remerciements}

Les auteurs remercient l'ARES-CCD pour l'appui financier à travers la bourse postdoctorale Elan octroyée à Y. Useni Sikuzani et S. Cabala Kaleba.

\section{Bibliographie}

Amisi M.Y. et al., 2008. Applicabilité de la théorie de perception de Kevin Lynch à une ville du tiers monde. Ann. Faculté Sci. Agron. Univ. Lubumbashi, 1(2), 7177. 
André M., Mahy G., Lejeune P. \& Bogaert J., 2014. Vers une synthèse de la conception et d'une définition des zones dans le gradient urbain-rural. Biotechnol. Agron. Soc. Environ., 18(1), 61-74.

Bamba I., Yedmel M.S. \& Bogaert J., 2010. Effets des routes et des villes sur la forêt dense dans la province orientale de la République Démocratique du Congo. Eur. J. Sci. Res., 43(3), 417-429.

Banza C.L.N. et al., 2009. High human exposure to cobalt and other metals in Katanga, a mining area of the Democratic Republic of Congo. Environ. Res., 109, 745752, doi: 10.1016/j.envres.2009.04.012

Barima Y.S.S. et al., 2009. Dynamique paysagère en milieu de transition forêt-savane ivoirienne. Bois For. Trop., 299(1), 15-25.

Bogaert J. \& Mahamane A., 2005. Écologie du paysage : cibler la configuration et l'échelle spatiale. Ann. Sci. Agron. Bénin, 7, 39-68.

Bogaert J., Biloso A., Vranken I. \& André M., 2015. Periurban dynamics: landscape ecology perspectives. In: Bogaert J. \& Halleux J.M., eds. Territoires périurbains : développement, enjeux et perspectives dans les pays $d u$ sud. Gembloux, Belgique: Les Presses agronomiques de Gembloux, 63-73.

Boisson S. et al., 2015. Concilier la phytostabilisation des sols pollués avec la conservation de la flore cupro-cobalticole dans la région de Lubumbashi : une stratégie pour valoriser les écosystèmes extrêmes ? In : Bogaert J. \& Halleux J.M., eds. Territoires périurbains : développement, enjeux et perspectives dans les pays du sud. Gembloux, Belgique : Les Presses agronomiques de Gembloux, 129-140.

Bruneau J.C. \& Pain M., 1990. Atlas de Lubumbashi. Nanterre, France : Université Paris X, 133.

Bryceson D. \& MacKinnon M., 2012. Eureka and beyond: mining's impact on African urbanization. J. Cont. Afr. Stud., 30(4), 513-537, doi: 10.1080/02589001.2012.719376

Caloz R. \& Collet C., 2001. Précis de télédétection : traitements numériques d'images de télédétection. Volume 3. Québec, Canada : Presses de 1'Université du Québec.

Cheyns K. et al., 2014. Pathways of human exposure to cobalt in Katanga, a mining area of the D.R. Congo. Sci. Total Environ., 490, 313-321, doi: 10.1016/j. scitotenv.2014.05.014

Ettler V., 2016. Soil contamination near non-ferrous metal smelters: a review. Appl. Geochem., 64, 56-74, doi: 10.1016/j.apgeochem.2015.09.020

Groupe Huit, 2009. Élaboration du plan urbain de référence de Lubumbashi. Rapport final. RD Congo: BEAU, Ministère des Infrastructures, travaux publics et reconstruction.

Gwebu T.D., 2012. Botswana's mining path to urbanization and poverty alleviation. J. Cont. Afr. Stud., 30(4), 611630, doi: 10.1080/02589001.2012.724868
Halleux J.-M., 2017. Le rôle du paradigme de l'accès à l'espace urbain dans la gestion de l'extension spatiale des villes congolaises. In : Actes du Colloque International "Architecture, urbanisme, paysage \& développement territorial: enjeux dans la société congolaise », 14 juillet 2017, Lubumbashi, RD Congo.

Havyarimana F. et al., 2018. La contribution des camps des déplacés à la dynamique paysagère au Sud et au Sud-est du Burundi. Tropicultura, 36(2), 243-257.

Joshi P.K. et al., 2006. Assessing areas deforested by coal mining activities through satellite remote sensing images and GIS in parts of Korba, Chattisgarh. J. Indian Soc. Remote Sens., 34(4), 415-421.

Khalil A., Hanich L., Hakkou R. \& Lepage M., 2014. GIS-based environmental database for assessing the mine pollution: a case study of an abandoned mine site in Morocco. J. Geochem. Explor., 144, 468-477, doi: 10.1016/j.gexplo.2014.03.023

Landis J.R. \& Koch G.G., 1977. The measurement of observer agreement for categorical data. Biometrics, 33(1), 159-174.

Leblanc M. \& Malaisse F., 1978. Lubumbashi, un écosystème urbain tropical. Université Nationale du Zaïre, Centre International de Sémiologie.

Leteinturier B., Baker A.J.M. \& Malaisse F., 1999. Early stages of natural revegetation of metalliferous mine workings in South Central Africa: a preliminary survey. Biotechnol. Agron. Soc. Environ., 3(1), 28-41.

Leteinturier B., Laroche J., Matera J. \& Malaisse F., 2001. Reclamation of lead/zinc processing wastes at Kabwe, Zambia: a phytogeochemical approach. S. Afr. J. Sci., 97, 1-4.

Maconachie R., 2012. Diamond mining, urbanization and social transformation in Sierra Leone. J. Cont. Afr. Stud., 30(4), 705-723, doi: 10.1080/02589001.2012.724872

Malaisse F., 2010. How to live and survive in Zambezian open forest (Miombo ecoregion). Gembloux, Belgium: Les Presses agronomiques de Gembloux.

Mama A., Sinsin B., De Cannière C. \& Bogaert J., 2013. Anthropisation et dynamique des paysages en zone soudanienne au nord du Bénin. Tropicultura, 31(1), 78-88.

Mas J.F., 2000. Une revue des méthodes et des techniques de télédétection du changement. Can. J. Remote Sens., 26(4), 349-362, doi : 10.1080/07038992.2000.10874785

Masse A., Ducrot D. \& Marthon P., 2012. Evaluation of supervised classification by class and classification characteristics. In: Algorithms and technologies for multispectral, hyperspectral, and ultraspectral imagery XVIII (Vol. 8390, p. 83902R). International Society for Optics and Photonics.

Mees F. et al., 2013. Concentrations and forms of heavy metals around two ore processing sites in Katanga, Democratic Republic of Congo. J. Afr. Earth Sci., 77, 22-30, doi: 10.1016/j.jafrearsci.2012.09.008 
Mpundu M.M., Useni S.Y., Nyembo K.L. \& Colinet G., 2014. Effets d'amendements carbonatés sur la culture de deux légumes sur sol contaminé à Lubumbashi (RD Congo). Biotechnol. Agron. Soc. Environ., 18(3), 367375 .

Mujinya B.B. et al., 2014. Spatial patterns and morphology of termite (Macrotermes falciger) mounds in the Upper Katanga, DR Congo. Catena, 114, 97-106, doi: 10.1016/j.catena.2013.10.015

Munyemba K.F. et al., 2008. Occupation des sols dans le cône de pollution à Lubumbashi. Ann. Faculté Sci. Agron. Univ. Lubumbashi, 1(2), 19-25.

Munyemba K.F. \& Bogaert J., 2014. Anthropisation et dynamique de l'occupation du sol dans la région de Lubumbashi de 1956 à 2009. E-revue UNILU, 1, 3-23.

Musa H.D. \& Jiya S.N., 2011. An assessment of mining activities impact on vegetation in Bukuru Jos Plateau State Nigeria using normalized differential vegetation index. J. Sustainable Dev ., 4(6), 150-160, doi: 10.5539/ jsd.v4n6p 150

Mususa P., 2012. Mining, welfare and urbanization: the wavering urban character of Zambia's Copperbelt. J. Cont. Afr. Stud., 30(4), 571-587, doi: 10.1080/02589001.2012.724873

Mwitwa J., German L., Muimba-Kankolongo A. \& Puntondewo A., 2012. Governance and sustainability challenges in landscape shaped by mining: miningforestry linkages and impacts in the copper belt of Zambia and the DR Congo. For. Policy Econ., 25, 19-30, doi: 10.1016/j.forpol.2012.08.001

Narendrula R., Nkongolo K.K. \& Beckett P., 2012. Comparative soil metal analyses in Sudbury (Ontario, Canada) and Lubumbashi (Katanga, DR-Congo). Bull. Environ. Contam. Toxicol., 88, 187-192, doi: 10.1007/ s00128-011-0485-7

Nkuku K.C. \& Rémon M., 2006. Stratégies de survie à Lubumbashi (R-D Congo). Enquête sur 14000 ménages urbains. Paris : l'Harmattan.

Panagos P., Van Liedekerke M., Yigini Y.\& Montanarella L., 2013. Contaminated sites in Europe: review of the current situation based on data collected through a European network. J. Environ. Public Health, 2013, 1-11, doi: 10.1155/2013/158764

Petropoulos G.P., Partsinevelos P. \& Mitraka Z., 2013. Change detection of surface mining activity and reclamation based on a machine learning approach of multitemporal Landsat TM imagery. Geocarto International, 28(4), 323-342, doi: 10.1080/10106049.2012.706648

Pontius R.G. \& Millones M., 2011. Death to Kappa: birth of quantity disagreement and allocation disagreement for accuracy assessment. Int. J. Remote Sens., 32(15), 4407-4429, doi: 10.1080/01431161.2011.552923

Pourret O. et al., 2016. Assessment of soil metal distribution and environmental impact of mining in Katanga (Democratic Republic of Congo). Appl. Geochem., 64, 43-55, doi: 10.1016/j.apgeochem.2015.07.012

Rodrigues C.U.\& Tavares A.P., 2012. Angola's planned and unplanned urban growth: diamond mining towns in the Lunda Provinces. J. Cont. Afr. Stud., 30(4), 687-703, doi: 10.1080/02589001.2012.724874

Seto K.C. \& Fragkias M., 2005. Quantifying spatiotemporal patterns of urban land-use change in four cities of China with time series landscape metrics. Landscape Ecol., 20(7), 871-888, doi: 10.1007/s10980-005-5238-8.

Shutcha M.N. et al., 2015. Three years of phytostabilisation experiment of bare acidic soil extremely contaminated by copper smelting using plant biodiversity of metalrich soils in tropical Africa (Katanga, DR Congo). Ecol. Eng., 82, 81-90, doi: 10.1016/j.ecoleng.2015.04.062

Tembo B.D., Sichilongo K. \& Cernak J., 2006. Distribution of copper, lead, cadmium and zinc concentrations in soils around Kabwe town in Zambia. Chemosphere, 63, 497-501, doi: 10.1016/j.chemosphere.2005.08.002

Trefon T. \& Cogels S., 2007. Espaces périurbains d'Afrique centrale et gouvernance environnementale. Bruxelles : Université libre de Bruxelles.

UN-Habitat, 2014. The state of African cities 2014. Reimagining sustainable urban transition. United Nations, Human Settlements programs.

Useni S.Y. et al., 2017. Le rayon de déforestation autour de la ville de Lubumbashi (Haut-Katanga, RD Congo) : synthèse. Tropicultura, 35(3), 215-221.

Useni S.Y. et al., 2018. Caractérisation de la croissance spatiale urbaine de la ville de Lubumbashi (HautKatanga, R.D. Congo) entre 1989 et 2014. Tropicultura, 36(1), 99-108.

Vranken I. et al., 2013. The spatial footprint of the nonferrous mining industry in Lubumbashi. Tropicultura, 31(1), 20-27.

Weerts A., 2015. Les sites miniers en République Démocratique du Congo: conflits et développement autourd'un espacehybride.In :Bogaert J.\&Halleux J.M., eds. Territoires périurbains : développement, enjeux et perspectives dans les pays du sud. Gembloux, Belgique : Les Presses agronomiques de Gembloux, 75-81. 
Annexe 1. Matrice de confusion pour l'évaluation de la performance des classifications supervisées (avant reclassification) des images SPOT de 1989 (a), 1996 (b), 2002 (c), 2005 (d), 2008 (e) et 2014 (f) - Confusion matrix for evaluating the performance of supervised classifications (before grouping of vegetation subclasses) of SPOT images of 1989 (a), 1996 (b), $2002(\boldsymbol{c}), 2005(\boldsymbol{d}), 2008(\boldsymbol{e})$ and $2014(\boldsymbol{f})$.

\begin{tabular}{|c|c|c|c|c|c|c|c|c|c|c|}
\hline \multirow{2}{*}{$\begin{array}{l}\text { a. Données de } \\
\text { référence }\end{array}$} & \multicolumn{10}{|c|}{ Données classifiées } \\
\hline & Terril & Eau & Marécage & Sol nu & Champs & Forêts & Savanes & Savanes herbeuses & Bâti & Total \\
\hline Terril & 20 & 0 & 0 & 0 & 0 & 0 & 0 & 0 & 0 & 20 \\
\hline Eau & 0 & 20 & 0 & 0 & 0 & 0 & 0 & 0 & 0 & 20 \\
\hline Marécage & 0 & 0 & 120 & 0 & 0 & 0 & 0 & 0 & 0 & 120 \\
\hline Sol nu & 0 & 0 & 0 & 99 & 0 & 0 & 0 & 6 & 15 & 120 \\
\hline Champs & 0 & 0 & 0 & 4 & 39 & 0 & 2 & 15 & 0 & 60 \\
\hline Forêts & 0 & 0 & 0 & 0 & 0 & 58 & 1 & 1 & 0 & 60 \\
\hline Savanes & 0 & 0 & 0 & 0 & 0 & 3 & 109 & 8 & 0 & 120 \\
\hline Savanes herbeuses & 0 & 0 & 0 & 8 & 8 & 0 & 0 & 104 & 0 & 120 \\
\hline Bâti & 0 & 0 & 0 & 11 & 0 & 0 & 0 & 0 & 109 & 120 \\
\hline Total & 20 & 20 & 120 & 122 & 47 & 61 & 112 & 134 & 124 & 760 \\
\hline
\end{tabular}

Précision globale : 89,2 \% ; Indice de Kappa : 87,5\%

\begin{tabular}{lllllllllll}
\hline $\begin{array}{l}\text { b. Données de } \\
\text { référence }\end{array}$ & \multicolumn{9}{l}{ Données classifiées } \\
\cline { 2 - 10 } & Terril & Eau & Marécage & Sol nu & Champs & Forêts & Savanes & Savanes herbeuses & Bâti & Total \\
\hline Terril & 20 & 0 & 0 & 0 & 0 & 0 & 0 & 0 & 0 & 20 \\
Eau & 0 & 20 & 0 & 0 & 0 & 0 & 0 & 0 & 0 & 20 \\
Marécage & 0 & 0 & 120 & 0 & 0 & 0 & 0 & 0 & 0 & 120 \\
Sol nu & 0 & 0 & 0 & 102 & 0 & 0 & 0 & 15 & 3 & 120 \\
Champs & 0 & 0 & 0 & 9 & 42 & 0 & 0 & 9 & 0 & 60 \\
Forêts & 0 & 0 & 0 & 0 & 0 & 53 & 5 & 2 & 0 & 60 \\
Savanes & 0 & 0 & 0 & 0 & 0 & 3 & 116 & 1 & 0 & 120 \\
Savanes herbeuses & 0 & 0 & 0 & 14 & 0 & 0 & 7 & 99 & 0 & 120 \\
Bâti & 0 & 0 & 0 & 12 & 0 & 0 & 0 & 0 & 108 & 120 \\
Total & 20 & 20 & 120 & 137 & 42 & 56 & 128 & 126 & 111 & 760 \\
\hline
\end{tabular}

Précision globale : 89,5\% ; Indice de Kappa : 87,7\%

\begin{tabular}{lllllllllll}
\hline \multirow{2}{*}{$\begin{array}{l}\text { c. Données de } \\
\text { référence }\end{array}$} & \multicolumn{7}{l}{ Données classifiées } \\
\cline { 2 - 10 } & Terril & Eau & Marécage & Sol nu & Champs & Forêts & Savanes & Savanes herbeuses & Bâti & Total \\
\hline Terril & 20 & 0 & 0 & 0 & 0 & 0 & 0 & 0 & 0 & 20 \\
Eau & 0 & 20 & 0 & 0 & 0 & 0 & 0 & 0 & 0 & 20 \\
Marécage & 0 & 0 & 120 & 0 & 0 & 0 & 0 & 0 & 0 & 120 \\
Sol nu & 0 & 0 & 0 & 98 & 0 & 0 & 0 & 15 & 7 & 120 \\
Champs & 0 & 0 & 0 & 11 & 40 & 0 & 0 & 9 & 0 & 60 \\
Forêts & 0 & 0 & 0 & 0 & 0 & 49 & 11 & 0 & 0 & 60 \\
Savanes & 0 & 0 & 0 & 0 & 0 & 3 & 116 & 1 & 0 & 120 \\
Savanes herbeuses & 0 & 0 & 0 & 14 & 0 & 0 & 7 & 99 & 0 & 120 \\
Bâti & 0 & 0 & 0 & 6 & 0 & 0 & 0 & 0 & 114 & 120 \\
Total & 20 & 20 & 120 & 129 & 40 & 52 & 134 & 124 & 121 & 760 \\
\hline
\end{tabular}

Précision globale : 88,9\% ; Indice de Kappa : 87,1\% 
Annexe 1 (suite). Matrice de confusion pour l'évaluation de la performance des classifications supervisées (avant reclassification) des images SPOT de 1989 (a), 1996 (b), 2002 (c), 2005 (d), 2008 (e) et 2014 (f) - Confusion matrix for evaluating the performance of supervised classifications (before grouping of vegetation subclasses) of SPOT images of 1989 (a), $1996(\boldsymbol{b}), 2002(\boldsymbol{c}), 2005(\boldsymbol{d}), 2008(\boldsymbol{e})$ and $2014(\boldsymbol{f})$.

\begin{tabular}{|c|c|c|c|c|c|c|c|c|c|c|}
\hline \multirow{2}{*}{$\begin{array}{l}\text { d. Données de } \\
\text { référence }\end{array}$} & \multicolumn{10}{|c|}{ Données classifiées } \\
\hline & Terril & Eau & Marécage & Sol nu & Champs & Forêts & Savanes & Savanes herbeuses & Bâti & Total \\
\hline Terril & 20 & 0 & 0 & 0 & 0 & 0 & 0 & 0 & 0 & 20 \\
\hline Eau & 0 & 20 & 0 & 0 & 0 & 0 & 0 & 0 & 0 & 20 \\
\hline Marécage & 0 & 0 & 120 & 0 & 0 & 0 & 0 & 0 & 0 & 120 \\
\hline Sol nu & 0 & 0 & 0 & 101 & 5 & 0 & 0 & 11 & 3 & 120 \\
\hline Champs & 0 & 0 & 0 & 0 & 60 & 0 & 0 & 0 & 0 & 60 \\
\hline Forêts & 0 & 0 & 0 & 0 & 0 & 52 & 8 & 0 & 0 & 60 \\
\hline Savanes & 0 & 0 & 0 & 0 & 0 & 4 & 114 & 2 & 0 & 120 \\
\hline Savanes herbeuses & 0 & 0 & 0 & 3 & 0 & 0 & 16 & 101 & 0 & 120 \\
\hline Bâti & 0 & 0 & 0 & 6 & 0 & 0 & 0 & 0 & 114 & 120 \\
\hline Total & 20 & 20 & 120 & 110 & 65 & 56 & 138 & 114 & 117 & 760 \\
\hline \multicolumn{11}{|c|}{ Précision globale : 92,4\% ; Indice de Kappa : 91,1\% } \\
\hline \multirow{2}{*}{$\begin{array}{l}\text { e. Données de } \\
\text { référence }\end{array}$} & \multicolumn{10}{|c|}{ Données classifiées } \\
\hline & Terril & Eau & Marécage & Sol nu & Champs & Forêts & Savanes & Savanes herbeuses & Bâti & Total \\
\hline Terril & 20 & 0 & 0 & 0 & 0 & 0 & 0 & 0 & 0 & 20 \\
\hline Eau & 0 & 20 & 0 & 0 & 0 & 0 & 0 & 0 & 0 & 20 \\
\hline Marécage & 0 & 0 & 120 & 0 & 0 & 0 & 0 & 0 & 0 & 120 \\
\hline Sol nu & 0 & 0 & 0 & 101 & 5 & 0 & 0 & 11 & 3 & 120 \\
\hline Champs & 0 & 0 & 0 & 0 & 60 & 0 & 0 & 0 & 0 & 60 \\
\hline Forêts & 0 & 0 & 0 & 0 & 0 & 55 & 5 & 0 & 0 & 60 \\
\hline Savanes & 0 & 0 & 0 & 0 & 0 & 8 & 112 & 0 & 0 & 120 \\
\hline Savanes herbeuses & 0 & 0 & 0 & 5 & 0 & 0 & 11 & 104 & 0 & 120 \\
\hline Bâti & 0 & 0 & 0 & 0 & 0 & 0 & 0 & 0 & 120 & 120 \\
\hline Total & 20 & 20 & 120 & 106 & 65 & 63 & 128 & 115 & 123 & 760 \\
\hline \multicolumn{11}{|c|}{ Précision globale : 93,7 \% ; Indice de Kappa : 92,7 \% } \\
\hline \multirow{2}{*}{$\begin{array}{l}\text { f. Données de } \\
\text { référence }\end{array}$} & \multicolumn{10}{|c|}{ Données classifiées } \\
\hline & $\underline{\text { Terril }}$ & Eau & Marécage & Sol nu & Champs & Forêts & Savanes & Savanes herbeuses & Bâti & Total \\
\hline Terril & 20 & 0 & 0 & 0 & 0 & 0 & 0 & 0 & 0 & 20 \\
\hline Eau & 0 & 20 & 0 & 0 & 0 & 0 & 0 & 0 & 0 & 20 \\
\hline Marécage & 0 & 0 & 120 & 0 & 0 & 0 & 0 & 0 & 0 & 120 \\
\hline Sol nu & 0 & 0 & 0 & 118 & 0 & 0 & 0 & 0 & 2 & 120 \\
\hline Champs & 0 & 0 & 0 & 0 & 60 & 0 & 0 & 0 & 0 & 60 \\
\hline Forêts & 0 & 0 & 0 & 0 & 0 & 60 & 0 & 0 & 0 & 60 \\
\hline Savanes & 0 & 0 & 0 & 0 & 0 & 0 & 120 & 0 & 0 & 120 \\
\hline Savanes herbeuses & 0 & 0 & 0 & 12 & 0 & 0 & 0 & 108 & 0 & 120 \\
\hline Bâti & 0 & 0 & 0 & 0 & 0 & 0 & 0 & 0 & 120 & 120 \\
\hline Total & 20 & 20 & 120 & 130 & 60 & 60 & 120 & 108 & 122 & 760 \\
\hline
\end{tabular}

